\title{
FUNGOS ASSOCIADOS A SEMENTES DE AÇAÍ: EFEITO DA TEMPERATURA E DO TEOR DE ÁGUA DAS SEMENTES DURANTE O ARMAZENAMENTO'
}

\author{
WALNICE MARIA OLIVEIRA DO NASCIMENTO², MARIA HELOISA DUARTE MORAES ${ }^{3}$
}

\begin{abstract}
RESUMO - O trabalho teve como objetivo verificar os efeitos do teor de água e da temperatura do ambiente de armazenamento na incidência de fungos e na germinação de sementes de açaí (Euterpe oleracea Mart.). Foram utilizadas sementes da cultivar BRS Pará, com teores de água de $43 \%, 37 \%, 30 \%, 26 \%, 21 \%$ e $12 \%$, que foram acondicionadas em sacos de polietileno, armazenadas sob temperaturas de $20^{\circ} \mathrm{C}, 15^{\circ} \mathrm{C}$ e $10^{\circ} \mathrm{C}$, durante 360 dias e submetidas a avaliações bimensais do teor de água, da germinação e da incidência de Penicillium sp., Aspergillus sp., Fusarium solani e Fusarium verticillioides. Foi adotado o delineamento experimental inteiramente casualizado com quatro repetições para o teste de germinação e cinco repetições para os testes de sanidade. Foi observada maior ocorrência de Penicillium sp., e Aspergillus sp em sementes com teores de água abaixo de $30 \%$ e mantidas nas temperaturas de $15^{\circ} \mathrm{C}$ e $20^{\circ} \mathrm{C}$. Enquanto, as maiores incidências de fungos do gênero Fusarium foram encontradas em sementes armazenadas com graus de umidade de $43 \%$ e $37 \%$ e mantidas em temperatura de $10{ }^{\circ} \mathrm{C}$.
\end{abstract}

Termos para indexação: Euterpe oleracea, sanidade, grau de umidade, conservação.

\section{FUNGI ASSOCIATED WITH AÇAI PALM SEEDS: EFFECT OF TEMPERATURE AND MOISTURE CONTENT ON SEEDS DURING STORAGE}

\begin{abstract}
The objective of the present study was to evaluate the effects of seed moisture content and storage temperature on the incidence of fungi and the germination of Euterpe oleracea seeds. Seeds of the BRS Para cultivar were used, with moisture contents of $43 \%, 37 \%, 30 \%, 26 \%$, $21 \%$ and $12 \%$, conditioned in polyethylene bags and stored under controlled temperatures of 20 ${ }^{\circ} \mathrm{C}, 15^{\circ} \mathrm{C}$ and $10^{\circ} \mathrm{C}$ for 360 days. The seeds were submitted to periodic evaluations of the moisture content, germination and the incidence of Penicillium sp., Aspergillus sp., Fusarium solani and Fusarium verticillioides. The experimental design was completely random, with four replicates for the germination test and five replicates for the health tests. A higher incidence of Penicillium sp. and Aspergillus sp. in seeds with a moisture content below $30 \%$ and at temperatures of $15{ }^{\circ} \mathrm{C}$ and $20{ }^{\circ} \mathrm{C}$ was observed whereas fungi of the genus Fusarium were found on seeds stored at $43 \%$ and $37 \% \mathrm{RH}$ and maintained at a temperature of $10{ }^{\circ} \mathrm{C}$.
\end{abstract}

Index terms: Euterpe oleracea, health, moisture content, conservation.

${ }^{1}$ Submetido em 03/04/2009. Aceito para publicação em 03/03/2011. Parte da Tese de Doutorado apresentada pelo primeiro autor à ESALQ/USP. Piracicaba (SP).

${ }^{2}$ Eng. Agrônoma, Dra. Pesquisadora da Embrapa Amazônia Oriental, Caixa Postal 48, CEP: 66095-100 Belém (PA) E-mail: walnice@cpatu. embrapa.br.

${ }^{3}$ Eng. Agrônoma, Dra. Departamento de Fitopatologia, Escola Superior de Agricultura "Luiz de Queiroz", Caixa Postal 9, 13418-900 Piracicaba (SP). E-mail: mhdmorae@esalq.usp.br. 


\section{INTRODUÇÃO}

A palmeira Euterpe oleracea (Mart.), conhecida como açaizeiro, tem se destacado economicamente pelo potencial mercadológico de seus produtos, representados, principalmente suco extraído do fruto e pelo palmito. É considerada espécie de grande importância para a população amazônica, devido à ocorrência natural na região, bem como pelo interesse econômico e social de seus produtos. As sementes do açaizeiro são admitidas como recalcitrantes (Carvalho et al., 1998; Nascimento e Silva, 2005) e, portanto, estão sujeitas à deterioração decorrente da secagem. Sementes recalcitrantes perdem a viabilidade quando o grau de umidade é reduzido abaixo de valores críticos, não podendo ser secas ou conservadas a temperaturas próximas de zero, pois ocorrem danos em nível celular. Esses aspectos fisiológicos limitam os ambientes para a conservação a médio e longo prazo (Roberts, 1973; King; Roberts, 1979; Chin, 1988). Em decorrência desses fatos a conservação das sementes recalcitrantes não pode ser efetuada pelos processos convencionais de armazenamento de sementes, que têm como pré-requisitos básicos, graus de umidade e temperaturas baixas para a adequada manutenção da qualidade. De modo geral, o armazenamento de sementes recalcitrantes deve proporcionar condições que previnam a perda de água, permitam o controle de micro-organismos patogênicos e mantenham o suprimento adequado de oxigênio (King e Roberts, 1980). Para Bonner (1990), a embalagem empregada deve ser impermeável ao vapor de água e, ao mesmo tempo, permeável aos gases para possibilitar as trocas gasosas necessárias à manutenção da viabilidade.

Portanto, o armazenamento de sementes com altos teores de água ainda é o principal método de conservação usado para sementes recalcitrantes. Esse método foi utilizado com relativo sucesso para sementes de seringueira (Hevea brasiliensis) (Chin et al., 1981; Cicero et al., 1986) arroz selvagem (Zizania palustris) (Probert e Longley, 1989), carvalho vermelho (Quercus rubor) (Gosling, 1989), Palmiteiro (Euterpe edulis) (Andrade, 2001) e camu-camuzeiro (Myrciaria dubia) (Gentil et al., 2004). Entretanto, se por um lado armazenar sementes com umidade elevada protege as espécies recalcitrantes, permitindo a atuação de mecanismos de reparo, por outro lado proporciona condições favoráveis ao desenvolvimento de micro-organismos. Já condições de baixas umidade e temperatura apenas favorecem a sobrevivência do inóculo, mas não estimula o desenvolvimento (Agarwal e
Sinclair, 1987). De acordo com Berjak (1996), os fungos associados às sementes, desempenham importante papel na deterioração pós-colheita das sementes dessas espécies.

Os fungos de campo, Colletotrichum spp., Fusarium spp., Phomopsis spp., se desenvolvem melhor e se mantêm viáveis em sementes com teores mais elevados de água, enquanto os de armazenamento, Aspergillus spp. e Penicillium spp., têm a habilidade de se desenvolver sem água livre, portanto em sementes mais secas. A atividade dos fungos de armazenamento ocorre quando o teor de água das sementes ultrapassa $13 \%$ e se acentua ao atingir valores superiores a 25\%. Em sementes de ingá (Inga uruguensis) armazenadas com $50 \%$ de água, houve acréscimos da atividade metabólica e da proliferação de Aspergillus spp. e de Penicillium sp., promovendo alterações no microambiente, identificadas pela elevação do grau de umidade das sementes (Bilia et al., 1999).

O trabalho objetivou verificar os efeitos do teor de água da semente e da temperatura do ambiente sobre a germinação e a incidência dos fungos Fusarium solani, Fusarium verticillioides, Aspergillus sp. e Penicillium sp., associados às sementes de açaí durante o armazenamento.

\section{MATERIAL E MÉTODOS}

Foram utilizadas sementes de açaí da cultivar BRS Pará. Imediatamente após a colheita, os frutos maduros foram submetidos à extração do epicarpo e mesocarpo em despolpadora mecânica, à lavagem para eliminação dos resíduos e à seleção manual objetivando o descarte daqueles mal formados ou parcialmente danificados. Para o armazenamento, foram utilizados como tratamentos sementes com sete diferentes teores de água, obtidos por meio da secagem em estufa com circulação forçada de ar.

As sementes com teores de água de: $43 \%, 37 \%, 30 \%$, $26 \%, 21 \%$ e $12 \%$ foram acondicionadas em sacos de polietileno transparente com dimensões de $30 \mathrm{~cm}$ x 20 $\mathrm{cm}$ e $0,1 \mathrm{~mm}$ de espessura (lacrados à quente), e depois armazenadas em câmaras com temperaturas controladas de $10 \pm 1{ }^{\circ} \mathrm{C}, 15 \pm 1{ }^{\circ} \mathrm{C}$ e $20 \pm 1{ }^{\circ} \mathrm{C}$. No início do armazenamento e bimensalmente, durante 360 dias, as sementes foram submetidas às avaliações discriminadas a seguir.

\section{Determinação do teor de água:}

Efetuada em estufa a $105 \pm 3{ }^{\circ} \mathrm{C}$, durante 24 horas, segundo as instruções das Regras para Análise de Sementes (Brasil, 1992), utilizando-se 10 sementes por repetição. Os resultados, expressos em porcentagem, foram calculados com base na massa úmida $(\mathrm{Bu})$. 


\section{Germinação:}

Realizada em areia umedecida com $70 \%$ de sua capacidade de retenção de água (Brasil, 1992), empregando 50 sementes por repetição, semeadas a $1 \mathrm{~cm}$ de profundidade, em ambiente protegido e desprovido de controles de temperatura e de umidade relativa do ar. Os resultados, obtidos 90 dias após a semeadura, foram expressos em porcentagem de plântulas normais.

\section{Sanidade das sementes:}

Foram empregadas cinco repetições de 10 sementes distribuídas, em caixas plásticas com dimensões de $11 \mathrm{~cm} \mathrm{x}$ $11 \mathrm{~cm} \times 3 \mathrm{~cm}$, sobre três folhas de papel de filtro previamente umedecidas comágua destilada. A seguir, as sementes foram incubadas à temperatura de $20^{\circ} \mathrm{C} \pm 2{ }^{\circ} \mathrm{C}$, por um período de sete dias, com regime alternado de 12 horas de luz N.U.V. (near ultraviolet) e 12 horas de escuro. Após sete dias de incubação, foi realizada avaliação da incidência dos fungos Fusarium solani, Fusarium verticillioides, Aspergillus sp. e Penicillium sp., individualmente em todas as sementes, por meio de observação das características morfológicas dos micro-organismos ao microscópio estereoscópico
(Lucca Filho, 1987) e por comparação com a literatura. Os resultados foram expressos em porcentagem.

Foi adotado o delineamento experimental inteiramente casualizado com sete tratamentos (graus de umidade da semente) antes do armazenamento e 21 tratamentos (sete graus de umidade $\mathrm{x}$ três temperaturas) em cada época de avaliação, durante o armazenamento. Para os dados obtidos durante o armazenamento, a análise de variância foi conduzida isoladamente em cada época de avaliação. Os dados em porcentagem foram transformados em arco seno da raiz quadrada de $x+\alpha / 100$. As médias foram comparadas pelo teste de Tukey ( $5 \%$ de probabilidade). Os dados do grau de umidade não foram submetidos à análise estatística.

\section{RESULTADOS E DISCUSSÃO}

A secagem progressiva intensificou o processo de deterioração das sementes, constatada pela redução da germinação. Quando a desidratação atingiu o teor de água de 15\%, o desempenho fisiológico foi anulado (Tabela 1).

TABELA 1. Teor de água, germinação e incidência de Penicillium sp., Aspergillus sp., Fusarium solani e Fusarium verticillioides, determinados em sementes de $E$. oleracea: valores médios obtidos antes do armazenamento.

\begin{tabular}{|c|c|c|c|c|c|}
\hline \multirow[b]{2}{*}{ Teor de água (\%) } & \multirow{2}{*}{ Germinação (\%) } & \multicolumn{4}{|c|}{ Incidência (\%) } \\
\hline & & Penicillium sp. & Aspergillus sp. & F. solani & F. verticillioides \\
\hline 43 & $92 \mathrm{a}^{1}$ & $00 \mathrm{~d}$ & $00 \mathrm{~b}$ & $54 \mathrm{a}$ & $12 \mathrm{ab}$ \\
\hline 37 & $88 \mathrm{a}$ & $00 \mathrm{~d}$ & $00 \mathrm{~b}$ & $60 \mathrm{a}$ & $10 a b$ \\
\hline 30 & $80 \mathrm{~b}$ & $00 \mathrm{~d}$ & $00 \mathrm{~b}$ & $50 \mathrm{a}$ & $16 \mathrm{a}$ \\
\hline 26 & $42 \mathrm{c}$ & $06 \mathrm{~d}$ & $00 \mathrm{~b}$ & $50 \mathrm{a}$ & $00 \mathrm{~b}$ \\
\hline 21 & $07 \mathrm{~d}$ & $14 \mathrm{ab}$ & $00 \mathrm{~b}$ & $22 \mathrm{~b}$ & $04 \mathrm{ab}$ \\
\hline 15 & $00 \mathrm{e}$ & $08 \mathrm{bc}$ & $08 \mathrm{a}$ & $04 \mathrm{c}$ & $02 \mathrm{ab}$ \\
\hline 12 & $00 \mathrm{e}$ & $20 \mathrm{a}$ & $00 \mathrm{~b}$ & $02 \mathrm{c}$ & $02 \mathrm{ab}$ \\
\hline
\end{tabular}

${ }^{1}$ Médias seguidas pela mesma letra, nas colunas, não diferem entre si pelo teste de Tukey a 5\% de probabilidade.

Os efeitos da dessecação sobre o desempenho fisiológico das sementes de açaí foram também verificados por Nascimento e Silva (2005), com a anulação da germinação quando a desidratação atingiu $15 \%$ de água. $\mathrm{O}$ avanço no processo de secagem afetou o desempenho das sementes, de forma que, aquelas que atingiram valores inferiores a 30\% de água, apresentaram acentuada redução da germinação e do vigor, comprometendo sua utilização.

A determinação do grau de umidade das sementes durante o período de armazenamento, indicou a manutenção dos tratamentos relativos aos graus de umidade nas temperaturas consideradas (Tabela 2). Entretanto, foram verificados aumentos no teor de água das sementes, com as maiores alterações no final do período de armazenamento, especialmente a partir de 300 dias, em sementes com $43 \%$ de água e em ambientes com temperaturas de $20{ }^{\circ} \mathrm{C}$ e $15^{\circ} \mathrm{C}$. Essa elevação no grau de umidade das sementes pode ser atribuída, principalmente, à deterioração das sementes, uma vez que não houve aumento na incidência de fungos, com exceção de Fusarium solani que teve sua incidência aumentada após 240 dias de armazenamento a $15{ }^{\circ} \mathrm{C}$. 
TABELA 2. Teor de água de sementes de $E$. oleracea, armazenadas por 360 dias a $20^{\circ} \mathrm{C}, 15^{\circ} \mathrm{C}$ e $10^{\circ} \mathrm{C}$.

\begin{tabular}{|c|c|c|c|c|c|c|c|}
\hline \multirow{2}{*}{\multicolumn{2}{|c|}{$\begin{array}{c}\text { Tratamento } \\
\text { (temperatura/teor de água) }\end{array}$}} & \multicolumn{6}{|c|}{ Período de armazenamento (dia) } \\
\hline & & 60 & 120 & 180 & 240 & 300 & 360 \\
\hline \multirow{7}{*}{$20^{\circ} \mathrm{C} /$} & $43 \%$ & 44,0 & 46,5 & 47,1 & 43,8 & 45,0 & 50,5 \\
\hline & $37 \%$ & 37,5 & 36,7 & 39,0 & 40,1 & 37,1 & 37,9 \\
\hline & $30 \%$ & 31,3 & 29,9 & 28,9 & 28,2 & 26,1 & 27,2 \\
\hline & $26 \%$ & 27,4 & 25,5 & 25,0 & 22,0 & 22,3 & 25,5 \\
\hline & $21 \%$ & 19,7 & 17,3 & 20,7 & 18,9 & 20,2 & 17,0 \\
\hline & $15 \%$ & 14,1 & 12,9 & 14,3 & 11,7 & 12,3 & 15,3 \\
\hline & $12 \%$ & 11,7 & 12,0 & 11,8 & 10,9 & 11,9 & 10,4 \\
\hline \multirow{7}{*}{$15^{\circ} \mathrm{C} /$} & $43 \%$ & 41,5 & 43,1 & 44,2 & 45,2 & 51,6 & 49,0 \\
\hline & $37 \%$ & 37,8 & 37,6 & 38,6 & 40,2 & 40,3 & 40,3 \\
\hline & $30 \%$ & 33,4 & 31,2 & 33,1 & 34,8 & 35,5 & 35,1 \\
\hline & $26 \%$ & 27,1 & 27,1 & 27,3 & 26,4 & 28,4 & 30,6 \\
\hline & $21 \%$ & 20,8 & 21,9 & 20,8 & 21,2 & 21,2 & 20,3 \\
\hline & $15 \%$ & 16,1 & 14,3 & 16,9 & 16,3 & 16,1 & 15,2 \\
\hline & $12 \%$ & 12,1 & 12,4 & 12,8 & 12,7 & 13,4 & 12,6 \\
\hline \multirow{7}{*}{$10^{\circ} \mathrm{C} /$} & $43 \%$ & 42,1 & 44,6 & 44,1 & 42,7 & 42,2 & 43,8 \\
\hline & $37 \%$ & 37,8 & 37,3 & 38,9 & 38,9 & 38,0 & 40,9 \\
\hline & $30 \%$ & 32,3 & 32,5 & 32,5 & 33,9 & 32,9 & 32,3 \\
\hline & $26 \%$ & 25,8 & 25,8 & 26,5 & 26,1 & 26,0 & 26,1 \\
\hline & $21 \%$ & 20,2 & 20,6 & 19,9 & 19,9 & 19,3 & 20,2 \\
\hline & $15 \%$ & 15,3 & 15,4 & 15,1 & 15,9 & 15,3 & 15,4 \\
\hline & $12 \%$ & 11,9 & 12,0 & 12,3 & 12,4 & 12,4 & 12,0 \\
\hline
\end{tabular}

Bilia et al. (1998) constataram variações no teor de água de sementes de Inga uruguensis, que foram atribuídas à deterioração das sementes e ao aumento da incidência de micro-organismos, os quais alteraram o microambiente. Alterações no teor de água de sementes de araucária, independentemente da embalagem e da temperatura utilizada para a conservação das sementes, foram identificadas também por (Tompsett 1984; Piriz Carrilo et al. 2003).

Os demais tratamentos apresentaram pouca variação em relação ao grau de umidade inicial. Sementes armazenadas em ambiente a $10^{\circ} \mathrm{C}$ apresentaram as menores variações no teor de água.

Os efeitos negativos da temperatura sobre $\mathrm{o}$ desempenho fisiológico das sementes de açaí foram proporcionais à redução da mesma. Os resultados obtidos para porcentagem de germinação durante o período do armazenamento nas temperaturas de $15{ }^{\circ} \mathrm{C}$ e $10{ }^{\circ} \mathrm{C}$ evidenciaram o rápido declínio do poder germinativo da espécie Euterpe oleracea (Tabela 3).

Foram identificadas quatro espécies de fungos associados às sementes: Penicillium sp, Aspergillus sp., Fusarium solani e Fusarium verticillioides. Antes do armazenamento a incidência de Penicillium sp. e Aspergillus sp., estava baixa para todos os teores de água na semente. Enquanto que, para os fungos Fusarium solani e $F$. verticillioide, foram constatadas as maiores porcentagens em sementes com teores de água acima de $26 \%$ (Tabela 1). Este resultado era esperado, pois segundo Agarwal e Sinclair (1987), fungos de campo se desenvolvem melhor em sementes com teor de água em equilíbrio com umidade relativa acima de $95 \%$, enquanto que para os de armazenamento a umidade relativa ideal fica entre $70 \%$ e $80 \%$. Isso resulta em sementes com teor de água de $24 \%$ a $25 \%$ e $13 \%$ a $18 \%$, respectivamente. 
TABELA 3. Germinação de sementes de E. oleracea: valores médios (\%) obtidos durante o armazenamento.

\begin{tabular}{|c|c|c|c|c|c|c|c|}
\hline \multirow{2}{*}{\multicolumn{2}{|c|}{$\begin{array}{c}\text { Tratamento } \\
\text { [temperatura }\left({ }^{\circ} \mathrm{C}\right) / \text { teor de água de \%] }\end{array}$}} & \multicolumn{6}{|c|}{ Período de armazenamento (dia) } \\
\hline & & 60 & 120 & 180 & 240 & 300 & 360 \\
\hline \multirow{7}{*}{$20^{\circ} \mathrm{C} /$} & $43 \%$ & $91 \mathrm{a}^{1}$ & $85 \mathrm{a}$ & $92 \mathrm{a}$ & $90 \mathrm{a}$ & $29 \mathrm{bc}$ & $00 \mathrm{~b}$ \\
\hline & $37 \%$ & $72 \mathrm{ab}$ & $68 \mathrm{~b}$ & $67 \mathrm{~b}$ & $82 \mathrm{a}$ & $40 \mathrm{~b}$ & $15 \mathrm{a}$ \\
\hline & $30 \%$ & $30 \mathrm{e}$ & $00 \mathrm{f}$ & $12 \mathrm{~d}$ & $12 \mathrm{c}$ & $00 \mathrm{~d}$ & $00 \mathrm{~b}$ \\
\hline & $26 \%$ & $25 \mathrm{e}$ & $00 \mathrm{f}$ & $00 \mathrm{e}$ & $00 \mathrm{e}$ & $00 \mathrm{~d}$ & $00 \mathrm{~b}$ \\
\hline & $21 \%$ & $00 \mathrm{f}$ & $00 \mathrm{f}$ & $00 \mathrm{e}$ & $00 \mathrm{e}$ & $00 \mathrm{~d}$ & $00 \mathrm{~b}$ \\
\hline & $15 \%$ & $00 \mathrm{f}$ & $00 \mathrm{f}$ & $00 \mathrm{e}$ & $00 \mathrm{e}$ & $00 \mathrm{~d}$ & $00 \mathrm{~b}$ \\
\hline & $12 \%$ & $00 \mathrm{f}$ & $00 \mathrm{f}$ & $00 \mathrm{e}$ & $00 \mathrm{e}$ & $00 \mathrm{~d}$ & $00 \mathrm{~b}$ \\
\hline \multirow{7}{*}{$15^{\circ} \mathrm{C} /$} & $43 \%$ & 41 cde & $18 \mathrm{e}$ & $00 \mathrm{e}$ & $00 \mathrm{e}$ & $00 \mathrm{~d}$ & $00 \mathrm{~b}$ \\
\hline & $37 \%$ & $69 a b$ & $50 \mathrm{c}$ & $57 \mathrm{~b}$ & $58 \mathrm{~b}$ & $63 \mathrm{a}$ & $14 \mathrm{a}$ \\
\hline & $30 \%$ & $55 \mathrm{bcd}$ & $36 \mathrm{~d}$ & $21 \mathrm{c}$ & $06 \mathrm{~d}$ & $22 \mathrm{c}$ & $00 \mathrm{~b}$ \\
\hline & $26 \%$ & $34 \mathrm{de}$ & $00 \mathrm{f}$ & $00 \mathrm{e}$ & $00 \mathrm{e}$ & $00 \mathrm{~d}$ & $00 \mathrm{~b}$ \\
\hline & $21 \%$ & $00 \mathrm{f}$ & $00 \mathrm{f}$ & $00 \mathrm{e}$ & $00 \mathrm{e}$ & $00 \mathrm{~d}$ & $00 \mathrm{~b}$ \\
\hline & $15 \%$ & $00 \mathrm{f}$ & $00 \mathrm{f}$ & $00 \mathrm{e}$ & $00 \mathrm{e}$ & $00 \mathrm{~d}$ & $00 \mathrm{~b}$ \\
\hline & $12 \%$ & $00 \mathrm{f}$ & $00 \mathrm{f}$ & $00 \mathrm{e}$ & $00 \mathrm{e}$ & $00 \mathrm{~d}$ & $00 \mathrm{~b}$ \\
\hline \multirow{7}{*}{$10^{\circ} \mathrm{C} /$} & $43 \%$ & $32 \mathrm{de}$ & $00 \mathrm{f}$ & $00 \mathrm{e}$ & $00 \mathrm{e}$ & $00 \mathrm{~d}$ & $00 \mathrm{~b}$ \\
\hline & $37 \%$ & $12 \mathrm{f}$ & $00 \mathrm{f}$ & $10 \mathrm{~d}$ & $00 \mathrm{e}$ & $00 \mathrm{~d}$ & $00 \mathrm{~b}$ \\
\hline & $30 \%$ & $63 \mathrm{abc}$ & $00 \mathrm{f}$ & $00 \mathrm{~d}$ & $00 \mathrm{e}$ & $00 \mathrm{~d}$ & $00 \mathrm{~b}$ \\
\hline & $26 \%$ & $32 \mathrm{de}$ & $00 \mathrm{f}$ & $00 \mathrm{e}$ & $00 \mathrm{e}$ & $00 \mathrm{~d}$ & $00 \mathrm{~b}$ \\
\hline & $21 \%$ & $00 \mathrm{f}$ & $00 \mathrm{f}$ & $00 \mathrm{e}$ & $00 \mathrm{e}$ & $00 \mathrm{~d}$ & $00 \mathrm{~b}$ \\
\hline & $15 \%$ & $00 \mathrm{f}$ & $00 \mathrm{f}$ & $00 \mathrm{e}$ & $00 \mathrm{e}$ & $00 \mathrm{~d}$ & $00 \mathrm{~b}$ \\
\hline & $12 \%$ & $00 \mathrm{f}$ & $00 \mathrm{f}$ & $00 \mathrm{e}$ & $00 \mathrm{e}$ & $00 \mathrm{~d}$ & $00 \mathrm{~b}$ \\
\hline
\end{tabular}

${ }^{1}$ Médias seguidas pela mesma letra, nas colunas, não diferem entre si pelo teste de Tukey a 5\% de probabilidade.

A incidência dos considerados "fungos de armazenamento", aumentou no período de armazenamento nas sementes que apresentavam teor de água entre $26 \%$ e $15 \%$ sob $15{ }^{\circ} \mathrm{C}$ e $20{ }^{\circ} \mathrm{C}$. De acordo com Christensen e Kaufmann (1965) a temperatura alta, associada ao elevado grau de umidade das sementes, favorece o desenvolvimento desses fungos. A associação de fungos como Penicillium e Aspergillus, durante o armazenamento, pode ser altamente prejudicial, pois deprecia a qualidade inicial das sementes através da perda do poder germinativo. Considerando que as sementes de açaí com menos de $26 \%$ de água tiveram a germinação anulada, imediatamente após a secagem, não foi possível observar associação direta entre a qualidade fisiológica e a incidência de micro-organismos nas sementes armazenadas com graus de umidade entre $12 \%$ e $21 \%$ (Tabelas 4 e 5 ).

Durante o período de armazenamento, as sementes mantidas a $10{ }^{\circ} \mathrm{C}$ e a $15{ }^{\circ} \mathrm{C}$ tiveram a manutenção da incidência de Fusarium solani e $F$. verticillioides, a qual pode ser atribuída ao elevado teor de água (43\% e 37\%) com que as sementes foram armazenadas ou à deterioração das sementes submetidas à baixa temperatura (Tabelas 6 e 7). Entretanto, para as sementes com comportamento de ortodoxas, onde o armazenamento é feito com reduzido teor de água em temperatura baixa, geralmente, há redução de fungos durante o armazenamento (Machado, 2000). Em pesquisa desenvolvida com sementes de soja, inoculadas com patógenos e armazenadas em câmara fria à $10{ }^{\circ} \mathrm{C}$, após seis meses foi verificada a redução da incidência do Phomopsis sojae e Colletotricum dematium var. truncata (Galli et al., 2007). 
TABELA 4. Incidência de Penicillium sp. em sementes de E. oleracea: valores médios (\%) obtidos durante o armazenamento.

\begin{tabular}{|c|c|c|c|c|c|c|c|}
\hline \multirow{2}{*}{\multicolumn{2}{|c|}{$\begin{array}{c}\text { Tratamento } \\
\left.\text { [temperatura }\left({ }^{\circ} \mathrm{C}\right) / \text { teor de água } \%\right]\end{array}$}} & \multicolumn{6}{|c|}{ Período de armazenamento (dia) } \\
\hline & & 60 & 120 & 180 & 240 & 300 & 360 \\
\hline \multirow{7}{*}{$20^{\circ} \mathrm{C} /$} & $43 \%$ & $00 \mathrm{c}^{1}$ & $00 \mathrm{~d}$ & $00 \mathrm{c}$ & $00 \mathrm{~g}$ & $00 \mathrm{~d}$ & $00 \mathrm{f}$ \\
\hline & $37 \%$ & $00 \mathrm{c}$ & $02 \mathrm{~d}$ & $00 \mathrm{c}$ & $00 \mathrm{~g}$ & $00 \mathrm{~d}$ & $00 \mathrm{f}$ \\
\hline & $30 \%$ & $12 a b$ & $08 \mathrm{bc}$ & $00 \mathrm{c}$ & $18 \mathrm{bcde}$ & $30 \mathrm{c}$ & $16 \mathrm{de}$ \\
\hline & $26 \%$ & $16 \mathrm{a}$ & $14 \mathrm{bc}$ & $30 \mathrm{ab}$ & $42 \mathrm{ab}$ & $58 \mathrm{a}$ & $40 \mathrm{bc}$ \\
\hline & $21 \%$ & $06 \mathrm{abc}$ & $26 a b$ & $75 \mathrm{a}$ & $50 \mathrm{a}$ & $56 \mathrm{ab}$ & $50 \mathrm{ab}$ \\
\hline & $15 \%$ & $04 \mathrm{abc}$ & $16 \mathrm{bc}$ & $04 \mathrm{c}$ & 28 abcd & $36 \mathrm{abc}$ & $52 \mathrm{a}$ \\
\hline & $12 \%$ & $06 \mathrm{abc}$ & $34 \mathrm{a}$ & $08 \mathrm{bc}$ & 18 bcde & $24 \mathrm{c}$ & $32 \mathrm{~cd}$ \\
\hline \multirow{7}{*}{$15^{\circ} \mathrm{C} /$} & $43 \%$ & $04 \mathrm{abc}$ & $02 \mathrm{~d}$ & $04 \mathrm{c}$ & $00 \mathrm{~g}$ & $00 \mathrm{~d}$ & $00 \mathrm{f}$ \\
\hline & $37 \%$ & $06 \mathrm{abc}$ & $00 \mathrm{~d}$ & $00 \mathrm{c}$ & $00 \mathrm{~g}$ & $00 \mathrm{~d}$ & $00 \mathrm{f}$ \\
\hline & $30 \%$ & $04 \mathrm{abc}$ & $10 \mathrm{bc}$ & $04 \mathrm{c}$ & $00 \mathrm{~g}$ & $00 \mathrm{~d}$ & $00 \mathrm{f}$ \\
\hline & $26 \%$ & $02 \mathrm{abc}$ & $04 \mathrm{~cd}$ & $16 \mathrm{bc}$ & $30 \mathrm{abc}$ & $40 a b$ & $20 \mathrm{de}$ \\
\hline & $21 \%$ & $04 a b c$ & $04 \mathrm{~cd}$ & $12 \mathrm{bc}$ & 26 abcd & $32 \mathrm{bc}$ & $38 \mathrm{bc}$ \\
\hline & $15 \%$ & $00 \mathrm{c}$ & $00 \mathrm{~d}$ & 08 cde & $02 \mathrm{~g}$ & $24 \mathrm{c}$ & $44 \mathrm{bc}$ \\
\hline & $12 \%$ & $00 \mathrm{c}$ & $06 \mathrm{~cd}$ & $04 \mathrm{c}$ & 04 efg & $00 \mathrm{~d}$ & $00 \mathrm{f}$ \\
\hline \multirow{7}{*}{$10^{\circ} \mathrm{C} /$} & $43 \%$ & $00 \mathrm{c}$ & $00 \mathrm{~d}$ & $00 \mathrm{c}$ & $02 \mathrm{~g}$ & $00 \mathrm{~d}$ & $00 \mathrm{f}$ \\
\hline & $37 \%$ & $00 \mathrm{c}$ & $02 \mathrm{~d}$ & $04 \mathrm{c}$ & $00 \mathrm{~g}$ & $00 \mathrm{~d}$ & $00 \mathrm{f}$ \\
\hline & $30 \%$ & $00 \mathrm{c}$ & $10 \mathrm{bc}$ & $00 \mathrm{c}$ & 06 efg & $00 \mathrm{~d}$ & $00 \mathrm{f}$ \\
\hline & $26 \%$ & $06 \mathrm{abc}$ & $14 \mathrm{bc}$ & $16 \mathrm{bc}$ & $10 \mathrm{defg}$ & $34 \mathrm{abc}$ & $16 \mathrm{de}$ \\
\hline & $21 \%$ & $04 \mathrm{abc}$ & $04 \mathrm{~cd}$ & $30 a b$ & $16 \mathrm{cdef}$ & $16 \mathrm{c}$ & 08 ef \\
\hline & $15 \%$ & $04 \mathrm{abc}$ & $02 \mathrm{~d}$ & $04 \mathrm{c}$ & 08 efg & $26 c$ & $14 \mathrm{e}$ \\
\hline & $12 \%$ & $00 \mathrm{c}$ & $00 \mathrm{~d}$ & $00 \mathrm{c}$ & $04 \mathrm{fg}$ & $32 \mathrm{bc}$ & $16 \mathrm{de}$ \\
\hline
\end{tabular}

\footnotetext{
${ }^{1}$ Médias seguidas pela mesma letra, nas colunas, não diferem entre si pelo teste de Tukey a 5\% de probabilidade.
} 
TABELA 5. Incidência de Aspergillus sp. em sementes de $E$. oleracea: valores médios (\%) obtidos durante o armazenamento.

\begin{tabular}{|c|c|c|c|c|c|c|c|}
\hline \multirow{2}{*}{\multicolumn{2}{|c|}{$\begin{array}{c}\text { Tratamento } \\
\text { (temperatura/teor de água) }\end{array}$}} & \multicolumn{6}{|c|}{ Período de armazenamento (dia) } \\
\hline & & 60 & 120 & 180 & 240 & 300 & 360 \\
\hline \multirow{7}{*}{$20^{\circ} \mathrm{C} /$} & $43 \%$ & $00 \mathrm{a}^{1}$ & $00 \mathrm{c}$ & $00 \mathrm{c}$ & $00 \mathrm{~b}$ & $00 \mathrm{e}$ & $00 \mathrm{f}$ \\
\hline & $37 \%$ & $00 \mathrm{a}$ & $00 \mathrm{c}$ & $00 \mathrm{c}$ & $00 \mathrm{~b}$ & $00 \mathrm{e}$ & $00 \mathrm{f}$ \\
\hline & $30 \%$ & $02 \mathrm{a}$ & $00 \mathrm{c}$ & $00 \mathrm{c}$ & $00 \mathrm{~b}$ & $00 \mathrm{e}$ & 04 ef \\
\hline & $26 \%$ & $04 \mathrm{a}$ & $00 \mathrm{c}$ & $02 \mathrm{c}$ & $00 \mathrm{~b}$ & $00 \mathrm{e}$ & 16 bcde \\
\hline & $21 \%$ & $04 \mathrm{a}$ & $32 \mathrm{a}$ & $40 \mathrm{a}$ & $34 \mathrm{a}$ & $52 \mathrm{a}$ & $22 \mathrm{abcd}$ \\
\hline & $15 \%$ & $00 \mathrm{a}$ & $04 \mathrm{bc}$ & $02 \mathrm{c}$ & $00 \mathrm{~b}$ & $24 \mathrm{bc}$ & 20 abcd \\
\hline & $12 \%$ & $00 \mathrm{a}$ & $08 \mathrm{~b}$ & $06 \mathrm{bc}$ & $02 \mathrm{~b}$ & $22 \mathrm{bc}$ & $32 \mathrm{ab}$ \\
\hline \multirow{7}{*}{$15^{\circ} \mathrm{C} /$} & $43 \%$ & $00 \mathrm{a}$ & $00 \mathrm{c}$ & $00 \mathrm{c}$ & $00 \mathrm{~b}$ & $00 \mathrm{e}$ & $00 \mathrm{f}$ \\
\hline & $37 \%$ & $00 \mathrm{a}$ & $00 \mathrm{c}$ & $00 \mathrm{c}$ & $00 \mathrm{~b}$ & $00 \mathrm{e}$ & $00 \mathrm{f}$ \\
\hline & $30 \%$ & $00 \mathrm{a}$ & $00 \mathrm{c}$ & $00 \mathrm{c}$ & $00 \mathrm{~b}$ & $00 \mathrm{e}$ & $34 \mathrm{a}$ \\
\hline & $26 \%$ & $00 \mathrm{a}$ & $08 \mathrm{~b}$ & $14 \mathrm{bc}$ & $10 \mathrm{ab}$ & $34 \mathrm{ab}$ & $26 a b c$ \\
\hline & $21 \%$ & $00 \mathrm{a}$ & $00 \mathrm{c}$ & $12 \mathrm{bc}$ & $08 \mathrm{~b}$ & $26 \mathrm{bc}$ & $26 \mathrm{abcd}$ \\
\hline & $15 \%$ & $00 \mathrm{a}$ & $00 \mathrm{c}$ & $08 \mathrm{bc}$ & $00 \mathrm{~b}$ & $18 \mathrm{bc}$ & $26 \mathrm{abcd}$ \\
\hline & $12 \%$ & $00 \mathrm{a}$ & $00 \mathrm{c}$ & $02 \mathrm{c}$ & $00 \mathrm{~b}$ & $10 \mathrm{cde}$ & $12 \mathrm{cde}$ \\
\hline \multirow{7}{*}{$10^{\circ} \mathrm{C} /$} & $43 \%$ & $00 \mathrm{a}$ & $00 \mathrm{c}$ & $00 \mathrm{c}$ & $00 \mathrm{~b}$ & $00 \mathrm{e}$ & $00 \mathrm{f}$ \\
\hline & $37 \%$ & $00 \mathrm{a}$ & $00 \mathrm{c}$ & $00 \mathrm{c}$ & $00 \mathrm{~b}$ & $00 \mathrm{e}$ & $00 \mathrm{f}$ \\
\hline & $30 \%$ & $00 \mathrm{a}$ & $00 \mathrm{c}$ & $00 \mathrm{c}$ & $00 \mathrm{~b}$ & $00 \mathrm{e}$ & $00 \mathrm{f}$ \\
\hline & $26 \%$ & $04 \mathrm{a}$ & $06 \mathrm{bc}$ & $16 \mathrm{~b}$ & $08 \mathrm{~b}$ & $14 \mathrm{bcd}$ & $28 \mathrm{abc}$ \\
\hline & $21 \%$ & $02 \mathrm{a}$ & $00 \mathrm{c}$ & $00 \mathrm{c}$ & $04 \mathrm{~b}$ & $08 \mathrm{e}$ & $10 \mathrm{de}$ \\
\hline & $15 \%$ & $04 \mathrm{a}$ & $00 \mathrm{c}$ & $00 \mathrm{c}$ & $04 \mathrm{~b}$ & $02 \mathrm{e}$ & $00 \mathrm{f}$ \\
\hline & $12 \%$ & $02 \mathrm{a}$ & $00 \mathrm{c}$ & $00 \mathrm{c}$ & $05 \mathrm{~b}$ & 12 cde & $00 \mathrm{f}$ \\
\hline
\end{tabular}

${ }^{1}$ Médias seguidas pela mesma letra, nas colunas, não diferem entre si pelo teste de Tukey a 5\% de probabilidade. 
TABELA 6. Incidência de Fusarium solani em sementes de $E$. oleracea: valores médios (\%) obtidos durante o armazenamento.

\begin{tabular}{|c|c|c|c|c|c|c|c|}
\hline \multirow{2}{*}{\multicolumn{2}{|c|}{$\begin{array}{c}\text { Tratamento } \\
\text { (temperatura/teor de água) }\end{array}$}} & \multicolumn{6}{|c|}{ Período de armazenamento (dia) } \\
\hline & & 60 & 120 & 180 & 240 & 300 & 360 \\
\hline \multirow{7}{*}{$20^{\circ} \mathrm{C} /$} & $43 \%$ & $28 \mathrm{bcd}^{1}$ & 12 cde & 04 ef & $12 \mathrm{de}$ & $04 \mathrm{c}$ & $04 \mathrm{c}$ \\
\hline & $37 \%$ & 18 cde & 14 cde & $16 \mathrm{bcd}$ & $26 \mathrm{bcd}$ & $22 \mathrm{~b}$ & $08 \mathrm{c}$ \\
\hline & $30 \%$ & $26 \mathrm{bcd}$ & $00 \mathrm{e}$ & $00 \mathrm{f}$ & $00 \mathrm{f}$ & $06 \mathrm{c}$ & $00 \mathrm{c}$ \\
\hline & $26 \%$ & 04 ef & $00 \mathrm{e}$ & 02 ef & $00 \mathrm{f}$ & $00 \mathrm{c}$ & $00 \mathrm{c}$ \\
\hline & $21 \%$ & 04 ef & $00 \mathrm{e}$ & $00 \mathrm{f}$ & $00 \mathrm{f}$ & $00 \mathrm{c}$ & $00 \mathrm{c}$ \\
\hline & $15 \%$ & $00 \mathrm{f}$ & $00 \mathrm{e}$ & $00 \mathrm{f}$ & $00 \mathrm{f}$ & $00 \mathrm{c}$ & $00 \mathrm{c}$ \\
\hline & $12 \%$ & $00 \mathrm{f}$ & $08 \mathrm{de}$ & 02 ef & $00 \mathrm{f}$ & $00 \mathrm{c}$ & $00 \mathrm{c}$ \\
\hline \multirow{7}{*}{$15^{\circ} \mathrm{C} /$} & $43 \%$ & $46 \mathrm{a}$ & $74 \mathrm{a}$ & $28 \mathrm{bc}$ & $38 \mathrm{abc}$ & $62 \mathrm{a}$ & $68 \mathrm{a}$ \\
\hline & $37 \%$ & $40 \mathrm{abc}$ & $44 \mathrm{~b}$ & $32 \mathrm{~b}$ & $60 \mathrm{a}$ & $64 \mathrm{a}$ & $72 \mathrm{a}$ \\
\hline & $30 \%$ & 06 ef & $24 \mathrm{bcd}$ & $00 \mathrm{f}$ & $12 \mathrm{ef}$ & $16 \mathrm{~b}$ & $00 \mathrm{c}$ \\
\hline & $26 \%$ & 04 ef & $00 \mathrm{e}$ & 04 ef & 06 ef & $00 \mathrm{c}$ & $00 \mathrm{c}$ \\
\hline & $21 \%$ & 04 ef & $02 \mathrm{e}$ & $00 \mathrm{f}$ & $00 \mathrm{f}$ & $00 \mathrm{c}$ & $00 \mathrm{c}$ \\
\hline & $15 \%$ & $08 \mathrm{def}$ & $00 \mathrm{e}$ & $00 \mathrm{f}$ & $00 \mathrm{f}$ & $00 \mathrm{c}$ & $00 \mathrm{c}$ \\
\hline & $12 \%$ & $02 \mathrm{f}$ & $06 \mathrm{de}$ & $00 \mathrm{f}$ & $00 \mathrm{f}$ & $00 \mathrm{c}$ & $00 \mathrm{c}$ \\
\hline \multirow{7}{*}{$10^{\circ} \mathrm{C} /$} & $43 \%$ & $54 \mathrm{a}$ & $80 \mathrm{a}$ & $60 \mathrm{a}$ & $38 \mathrm{abc}$ & $56 \mathrm{a}$ & $52 \mathrm{~b}$ \\
\hline & $37 \%$ & $44 \mathrm{ab}$ & $36 \mathrm{bc}$ & 12 cde & $46 \mathrm{ab}$ & $24 \mathrm{~b}$ & $40 \mathrm{~b}$ \\
\hline & $30 \%$ & $22 \mathrm{bcd}$ & $38 \mathrm{bc}$ & $20 \mathrm{bc}$ & 08 ef & $00 \mathrm{c}$ & $00 \mathrm{c}$ \\
\hline & $26 \%$ & 04 ef & $02 \mathrm{e}$ & 14 cde & $00 \mathrm{f}$ & $00 \mathrm{c}$ & $00 \mathrm{c}$ \\
\hline & $21 \%$ & 04 ef & $00 \mathrm{e}$ & $00 \mathrm{f}$ & $00 \mathrm{f}$ & $00 \mathrm{c}$ & $00 \mathrm{c}$ \\
\hline & $15 \%$ & $16 \mathrm{de}$ & $04 \mathrm{de}$ & $00 \mathrm{f}$ & $00 \mathrm{f}$ & $00 \mathrm{c}$ & $00 \mathrm{c}$ \\
\hline & $12 \%$ & $06 \mathrm{def}$ & $00 \mathrm{e}$ & $04 \mathrm{def}$ & $00 \mathrm{f}$ & $00 \mathrm{c}$ & $00 \mathrm{c}$ \\
\hline
\end{tabular}

${ }^{1}$ Médias seguidas pela mesma letra, nas colunas, não diferem entre si pelo teste de Tukey a 5\% de probabilidade. 
TABELA 7. Incidência de Fusarium verticillioides em sementes de E. oleracea: valores médios (\%) obtidos durante o armazenamento.

\begin{tabular}{|c|c|c|c|c|c|c|c|}
\hline \multirow{2}{*}{\multicolumn{2}{|c|}{$\begin{array}{c}\text { Tratamento } \\
\text { (temperatura/teor de água) }\end{array}$}} & \multicolumn{6}{|c|}{ Período de armazenamento (dia) } \\
\hline & & 60 & 120 & 180 & 240 & 300 & 360 \\
\hline \multirow{7}{*}{$20^{\circ} \mathrm{C} /$} & $43 \%$ & $00 b^{1}$ & $04 \mathrm{~cd}$ & $16 \mathrm{bc}$ & $00 \mathrm{~d}$ & $02 \mathrm{c}$ & $00 \mathrm{~d}$ \\
\hline & $37 \%$ & $02 \mathrm{~b}$ & $04 \mathrm{~cd}$ & 06 cde & $00 \mathrm{~d}$ & $06 \mathrm{bc}$ & $00 \mathrm{~d}$ \\
\hline & $30 \%$ & $00 \mathrm{~b}$ & $00 \mathrm{~d}$ & $00 \mathrm{e}$ & $00 \mathrm{~d}$ & $00 \mathrm{c}$ & $00 \mathrm{~d}$ \\
\hline & $26 \%$ & $00 \mathrm{~b}$ & $00 \mathrm{~d}$ & $02 \mathrm{de}$ & $00 \mathrm{~d}$ & $00 \mathrm{c}$ & $00 \mathrm{~d}$ \\
\hline & $21 \%$ & $02 \mathrm{~b}$ & $00 \mathrm{~d}$ & $00 \mathrm{e}$ & $00 \mathrm{~d}$ & $00 \mathrm{c}$ & $00 \mathrm{~d}$ \\
\hline & $15 \%$ & $00 \mathrm{~b}$ & $00 \mathrm{~d}$ & $00 \mathrm{e}$ & $00 \mathrm{~d}$ & $00 \mathrm{c}$ & $00 \mathrm{~d}$ \\
\hline & $12 \%$ & $00 \mathrm{~b}$ & $00 \mathrm{~d}$ & $00 \mathrm{e}$ & $00 \mathrm{~d}$ & $00 \mathrm{c}$ & $00 \mathrm{~d}$ \\
\hline \multirow{7}{*}{$15^{\circ} \mathrm{C} /$} & $43 \%$ & $02 \mathrm{~b}$ & $00 \mathrm{~d}$ & $16 \mathrm{bc}$ & $28 \mathrm{a}$ & $02 \mathrm{c}$ & $08 \mathrm{bc}$ \\
\hline & $37 \%$ & $24 \mathrm{a}$ & $42 \mathrm{ab}$ & $22 \mathrm{~b}$ & $10 \mathrm{bcd}$ & $36 \mathrm{a}$ & $12 \mathrm{ab}$ \\
\hline & $30 \%$ & $00 \mathrm{~b}$ & $48 \mathrm{a}$ & $16 \mathrm{bc}$ & $14 a b c$ & $00 \mathrm{c}$ & $00 \mathrm{~d}$ \\
\hline & $26 \%$ & $06 \mathrm{~b}$ & $00 \mathrm{~d}$ & $00 \mathrm{e}$ & $04 \mathrm{~cd}$ & $00 \mathrm{c}$ & $00 \mathrm{~d}$ \\
\hline & $21 \%$ & $02 \mathrm{~b}$ & $00 \mathrm{~d}$ & $00 \mathrm{e}$ & $00 \mathrm{~d}$ & $00 \mathrm{c}$ & $00 \mathrm{~d}$ \\
\hline & $15 \%$ & $00 \mathrm{~b}$ & $00 \mathrm{~d}$ & $00 \mathrm{e}$ & $00 \mathrm{~d}$ & $00 \mathrm{c}$ & $00 \mathrm{~d}$ \\
\hline & $12 \%$ & $00 \mathrm{~b}$ & $00 \mathrm{~d}$ & $02 \mathrm{de}$ & $00 \mathrm{~d}$ & $00 \mathrm{c}$ & $00 \mathrm{~d}$ \\
\hline \multirow{7}{*}{$10^{\circ} \mathrm{C} /$} & $43 \%$ & $24 \mathrm{a}$ & $48 \mathrm{a}$ & $16 \mathrm{bc}$ & $12 \mathrm{bcd}$ & $10 \mathrm{~b}$ & $02 \mathrm{~cd}$ \\
\hline & $37 \%$ & $22 \mathrm{a}$ & $36 \mathrm{ab}$ & $34 \mathrm{a}$ & $18 \mathrm{abc}$ & $24 \mathrm{a}$ & $16 \mathrm{a}$ \\
\hline & $30 \%$ & $06 \mathrm{~b}$ & $08 \mathrm{c}$ & $12 \mathrm{bcd}$ & $22 \mathrm{ab}$ & $00 \mathrm{c}$ & $00 \mathrm{~d}$ \\
\hline & $26 \%$ & $02 \mathrm{~b}$ & $00 \mathrm{~d}$ & $00 \mathrm{e}$ & $00 \mathrm{~d}$ & $00 \mathrm{c}$ & $00 \mathrm{~d}$ \\
\hline & $21 \%$ & $00 \mathrm{~b}$ & $00 \mathrm{~d}$ & $00 \mathrm{e}$ & $00 \mathrm{~d}$ & $00 \mathrm{c}$ & $00 \mathrm{~d}$ \\
\hline & $15 \%$ & $00 \mathrm{~b}$ & $02 \mathrm{~d}$ & $00 \mathrm{e}$ & $00 \mathrm{~d}$ & $00 \mathrm{c}$ & $00 \mathrm{~d}$ \\
\hline & $12 \%$ & $00 \mathrm{~b}$ & $00 \mathrm{~d}$ & $00 \mathrm{e}$ & $00 \mathrm{~d}$ & $00 \mathrm{c}$ & $00 \mathrm{~d}$ \\
\hline
\end{tabular}

${ }^{1}$ Médias seguidas pela mesma letra, nas colunas, não diferem entre si pelo teste de Tukey a 5\% de probabilidade.

Não foi possível estabelecer relações entre a incidência de micro-organismos e a germinação das sementes de açaí, durante o período de armazenamento. Resultado similar foi obtido por Gentil, (2003) em sementes de camu-camu (Myrciaria dubia). Contudo, para as sementes de pupunha (Bactris gasipaes) CoatesBeckford e Chung (1987) relacionaram a incidência alta de Fusarium solani à redução da germinação e ao transporte do fungo pela semente.

A ocorrência de Penicillium sp. e Aspergillus sp foi maior em sementes com teores de água abaixo de $30 \%$ e mantidas nas temperaturas de $15{ }^{\circ} \mathrm{C}$ e $20^{\circ} \mathrm{C}$. Enquanto, as maiores incidências de fungos do gênero Fusarium foram encontradas em sementes armazenadas com graus de umidade de $43 \%$ e $37 \%$ e mantidas em temperatura de $10{ }^{\circ} \mathrm{C}$.

\section{CONCLUSÕES}

Para a conservação da qualidade das sementes de açaí, isto é, manter a viabilidade e evitar a infestação de fungos do gênero Fusarium, Aspergillus e Penicillum, o armazenamento deve ser feito em temperatura constante de $20{ }^{\circ} \mathrm{C}$ e com as sementes com teor de água 37 e $43 \%$.

\section{REFERÊNCIAS}

AGARWAL, V.K.; SINCLAIR, J.B. Principles of seed pathology, Florida: CRC Press, 1987. v.2, 168p.

ANDRADE, A.C.S. The effect of moisture content and temperature on the longevity of heart of palm seeds (Euterpe edulis). Seed Science and Technology, v.29, n.1, 
p.171-182, 2001.

BERJAK, P. The role of micro-organisms in deterioration during storage of recalcitrant and intermediate seeds. QUEDRAOGO, A.S.; POULSEN, K.; STUBSGAARD, F. (Ed.). In: WORKSHOP ON IMPROVED METHODS FOR HANDLING AND STORAGE OF INTERMEDIATE/ RECALCITRANT TROPICAL FLOREST TREE SEEDS. 1996: Rome, Proceedings... Rome: IPGRI. p.121-126. 1996.

BILIA, D.A.C.; MARCOS FILHO, J.; NOVEMBRE, A.D.L.C. Desiccation tolerance and storability of Inga uruguensis seed. Seed Science and Technology, v.27, n.1, p.77-89, 1999.

BILIA, D.A.C.; MARCOS FILHO. J.; NOVEMBRE, A.D.L.C. Conservação da qualidade fisiológica de sementes de Inga uruguensis Hook. Et Arn. Revista Brasileira de Sementes, v.20, n.1, p.48-54, 1998.

BONNER, F.T. Storage of seeds: potential and limitations for germoplasm conservation. Forest Ecology and Management, v.35, n.1, p.35-43, 1990.

BRASIL. Ministério da Agricultura e Reforma Agrária. Secretaria Nacional de Defesa Agropecuária. Departamento Nacional de Produção Vegetal. Coordenação de Laboratório Vegetal. Regras para análise de sementes. Brasília, DF, 1992. 365p.

CARVALHO, J.E.U.; NASCIMENTO, W.M.O.; MÜLLER, C.H. Características físicas e de germinação de sementes de espécies frutíferas nativas da Amazônia. Belém, PA: Embrapa-CPATU, 1998. 18p. (EmbrapaCPATU. Boletim de pesquisa, 203).

CHIN, H.F. AZIZ, M.; ANG, B.B.; HAMZAH, S. The effect of moisture and temperature on the ultrastructure and viability of seeds of Hevea brasiliensis. Seed Science and Technology, v.9, n.2, p.411-422, 1981.

CHIN, H.F. Recalcitrant seed: a status report. Rome: IPGRI, 1988. 18p.

CHRISTENSEN, C.M.; KAUFMANN, H.H. Deterioration of storage grains by fungi. Annual Review Phytopathology, v.3, n.1, p.69-84, 1965.

CICERO, S.M.; MARCOS FILHO, J.; TOLEDO, F.F. Efeitos do tratamento fungicida e de três ambientes de armazenamento sobre a conservação de sementes de seringueira. Anais da Escola Superior de Agricultura “Luiz de Queiroz", v.43, p.763-787, 1986.

COATES-BECKFORD, P.; CHUNG, P.C. A study of the germination, disease symptoms and fungi associated with pejibaye seeds. Seed Science and Technology, v.15, n.1, p.205-218, 1987.

GALLI, J.A.; PANIZI, R.C.; VIEIRA, R.D. Sobrevivência de patógenos associados a sementes de soja armazenadas durante seis meses. Revista Brasileira de Sementes, v.29, n.2, p.205-213, 2007.

GENTIL, D.F.O. Conservação de sementes de Myrciaria dubia (H.B.K.) McVAUGH. 2003. 41f. Tese (Doutorado em Fitotecnia) - Escola Superior de Agricultura "Luiz de Queiroz”, Universidade de São Paulo, Piracicaba, 2003.

GENTIL, D.F.O.; SILVA, W.R.; FERREIRA, S.A.N. Conservação de sementes de Myrciaria dubia (H.B.K.) McVaugh. Bragantia, v.63, n.3, p.421-430, 2004.

GOSLING, P.G. The effect of drying Quercus rubor acorns to different moisture contents, followed by storage, either with or without imbibitions. Forestry, v.62, n.1, p.41-50, 1989.

KING, M.W.; ROBERTS, E.H. The storage of recalcitrant seed: achievements and possible approaches. Rome: IPGRI, 1979. 96p.

KING, M.W.; ROBERTS, E.H. A strategy for future research into the storage of recalcitrant seeds. In: CHIN, H.F.; ROBERTS, E.H. (Ed.) Recalcitrant crop seeds. Kuala Lumpur: Tropical Press, 1980. cap.5, p.90-110.

LUCCA FILHO, O.A. Metodologia dos testes de sanidade de sementes. In: SOAVE, J.; WETZEL, M.M.V.S.(Ed.). Patologia de Sementes. Campinas: Fundação Cargill, 1987. cap. 10, p.276-298.

MACHADO, J.C. - Patologia de sementes: significado e atribuições. In: CARVALHO, N.M. de; NAKAGAWA, J. Sementes: ciência, tecnologia e produção.4.ed. Jaboticabal: FUNEP, 2000. p.522-588.

NASCIMENTO, W.M.O.; SILVA, W.R. Comportamento fisiológico de sementes de açaí (Euterpe oleracea Mart.) submetidas à desidratação. Revista Brasileira de Fruticultura, v.27, n.3, p.349-351, 2005.

PROBERT; R.J.; LONGLEY, P.L. Recalcitrant seeds 
storage physiology in three aquatic grasses (Zizania palustris, Spartina anglica and Porteresia coarctata). Annals of Botany, v.63, n.1, p.53-63, 1989.

PIRIZ CARILLO, V.; CHAVES, A.; FASSOLA, H.; MUGRIDGE, A. A refrigerated storage of seeds of Araucaria angustifólia (Bert.) O. Kuntze over a period of 24 months. Seed Science and Technology, v.31, n.2, p.411-429, 2003.

ROBERTS, E.H. Predicting the storage life of seeds. Seed Science and Technology, v.1, p.499-514, 1973.

TOMPSETT, P.B. The influence of a gaseous environment on the storage life of Araucaria husteeinii seeds. Annals of Botany, v.52, n.1, p.229-237, 1984. 